\title{
Particle sizing in concentrated suspensions by use of steady-state, continuous-wave photon-migration techniques
}

\author{
Huabei Jiang \\ Department of Physics and Astronomy, Clemson University, Clemson, South Carolina 29634-1911 \\ Guillermo Marquez and Lihong V. Wang \\ Biomedical Engineering Program, Texas A\&M University, College Station, Texas 77843-3120
}

Received October 14, 1997

\begin{abstract}
We demonstrate a new approach for determining particle-size distribution in concentrated suspensions from spectral measurement of isotropic scattering coefficients by use of steady-state, continuous-wave photonmigration techniques. Successful recovery of particle-size distribution for $\mathrm{TiO}_{2}$ suspensions in the form of log-normal functions is achieved through a regularized inverse algorithm, into which a synthesized scheme of Marquardt and Tikhonov regularizations has been incorporated. Our results for dense $\mathrm{TiO}_{2}$ suspensions with three different particle concentrations are in excellent agreement with the size distribution as measured with x-ray sedimentation. (C) 1998 Optical Society of America

OCIS codes: $\quad 350.4990,290.4210,170.5280$.
\end{abstract}

Particle sizing in nondilute suspensions is important in areas such as environmental remote sensing and online control of chemical- and pharmaceutical-based industrial processes. Among the existing particle-sizing techniques, optical methods such as dynamic light scattering and optical-turbidity or extinction-spectrum measurements have been particularly attractive because of their portability and potential for rapid measurement. ${ }^{1,2}$ Although researchers have used these optical techniques to obtain size information about scattering particles noninvasively, the use of these techniques is restricted to diluted suspensions and often requires extensive calibrations with the suspensions that they are intended to monitor.

To overcome the problems associated with the present optical techniques for particle sizing, Jiang et al. recently proposed a new optical method based on frequency-domain measurements of photon migration in scattering suspensions. ${ }^{3}$ Since this technique depends on multiply scattered light, it is particularly suitable for nondilute suspensions and has great potential for on-line process monitoring. In addition, the technique does not require calibrations with the suspensions.

However, the measurements that we conducted were based on a frequency-domain Ti:sapphire laser system, which does not provide a light beam with a wide spectrum range. Further, the procedures for optical data collection were relatively slow, since multiwavelength measurements were needed. In this Letter we experimentally demonstrate particle sizing in concentrated $\mathrm{TiO}_{2}$ suspensions for what we believe to be the first time, using cw-based photon-migration techniques. This method allows faster and simpler optical measurements than frequency-domain techniques, with the same accuracy of measurements of optical properties in a scattering medium. ${ }^{4-6}$ In addition, the cost of a cw-based system is lower than that of its frequency-domain counterpart. Using a regularized inverse algorithm, we reconstructed particle-size distributions of $\mathrm{TiO}_{2}$ suspensions with three different concentrations. The results are in excellent agreement with the size distribution as measured with x-ray sedimentation.

Particle-size distributions are obtained with a regularized inverse algorithm under Mie scattering theory, as reported in Ref. 3. Our approach casts particle sizing as an optimization problem in which the optimization parameters are coefficients in a probability function, such as an a priori log-normal or Gaussian distribution function. The details of our inverse algorithms can be found in Ref. 3; we present a brief overview here for context.

It is known that particle-size distribution in a multiple-scattering medium is correlated with the isotropic scattering coefficient of the medium by the following relationship ${ }^{7,8}$ :

$$
\begin{aligned}
(1-g) \mu_{s}(\lambda)= & \mu_{s}{ }^{\prime}(\lambda) \\
= & \int_{0}^{\infty} \frac{3 Q_{\text {scat }}(x, n, \lambda)[1-g(x, n, \lambda)]}{2 x} \\
& \times \phi f(x) \mathrm{d} x,
\end{aligned}
$$

where $(1-g) \mu_{s}$ is the isotropic scattering coefficient, $g$ is the mean cosine of the scattering angle from a single particle, $Q_{\text {scat }}$ is the scattering efficiency, $n$ is the refractive index of the medium, and $\lambda$ is the wavelength of light; $f(x)$ ( $x$ is the diameter of the particles) is the particle-volume size distribution, and $\phi$ is the total volume fraction of the particles. Both $g$ and $Q_{\text {scat }}$ can be computed with Mie theory. ${ }^{8}$

For concentrated suspensions, we usually have a priori knowledge about the number of modes and 
their size distributions. Generally, particulate suspensions can be characterized as Gaussian or log-normal distributions. We use log-normal distributions for $\mathrm{TiO}_{2}$ suspension in this study': $f(x)=$ $\exp \left[-(\ln x-a)^{2} / 2 b^{2}\right] / \sqrt{2 \pi} b x$, where parameters $a$ and $b$ denote the mean and the variance of the distribution, respectively. Using our regularized optimization methods, we obtain the following system of equations, which leads to an inverse problem solution ${ }^{3}$ :

$$
\left(\mathcal{T}^{T} \mathcal{T}+\alpha I\right) \Delta \zeta=\mathcal{T}^{T}\left[\left(\mu_{s}{ }^{\prime}\right)^{o}-\left(\mu_{s}^{\prime}\right)^{c}\right]
$$

where the Jacobian matrix $\mathcal{T}$ represents the sensitivity of isotropic scattering coefficients measured at multiple wavelengths, the elements of which are numerically computed with Eq. (1). I is the identity matrix, and $\alpha$ can be a scalar or a diagonal matrix. The vectors $\left(\mu_{s}^{\prime}\right)^{o}$ and $\left(\mu_{s}^{\prime}\right)^{c}$ contain the observed and the computed values of isotropic scattering coefficients at the wavelengths of interest, respectively. $\Delta \zeta$ is the vector that updates parameters $a, b$, and $c: \Delta \zeta=(\delta a, \delta b, \delta c)^{T}$ (we choose $\phi$ as the third parameter). Thus the basic idea in determining $a, b$, and $c$ is to measure the isotropic scattering coefficient of the suspensions at multiple wavelengths. Then the particle-sizing task is to perform the estimates [which are updated and improved through solution of Eq. (2)] of parameters $a, b$, and $c$ that are required for the measured scattering coefficients to be sustained. Inasmuch as the matrix $\mathcal{T} \mathcal{T}^{T}$ is known to be ill conditioned, ${ }^{2,3}$ a way to regularize or stabilize the decomposition of $\mathcal{T} \mathcal{T}^{T}$ is needed. We used a hybrid technique that synthesizes standard Marquardt and Tikhonov regularization schemes. ${ }^{3}$

The experimental system, shown schematically in Fig. 1, was based on oblique-incidence reflectrometry. ${ }^{4-6}$ White light (Oriel 75-W xenon arc lamp) was delivered, and the diffuse reflectance was collected with a fiber-optic probe made from black Delrin and 600- $\mu \mathrm{m}$-diameter, low-loss optical fibers. The source fiber was oriented at a $45^{\circ}$ angle of incidence, and the nine collection fibers, arranged in a linear array, collected the diffuse reflectance. To correct for slight variations in collection efficiency from one detection fiber to the next, we calculated a correction factor for each fiber based on a calibration procedure using standard phantoms. The detection system was composed of an imaging spectrograph (Oriel Multispec 257), a CCD camera (Princeton Instruments 1530P), and a personal computer that automatically recorded the spectrum of the collected light through the wavelength range $400-800 \mathrm{~nm}$. The spectral resolution of the measurements was $0.5 \mathrm{~nm}$.

The fiber probe was placed on the surface of each concentrated suspension. We attached an exposed x-ray film to the top of each suspension to approximate a matched boundary condition for diffusion theory. Four measurements were performed with the probe oriented at $0^{\circ}, 30^{\circ}, 60^{\circ}$, and $90^{\circ}$ with respect to an arbitrary reference orientation. The isotropic scattering spectra were evaluated for each diffuse reflectance measurement, and the results were averaged. The concentrated suspensions were mixtures of $\mathrm{TiO}_{2}$, India ink, and polyacrylamide gel, ${ }^{10}$ which produced $\mathrm{TiO}_{2}$ concentrations of $0.88,1.17$, and 1.46 wt \%, re- spectively. Each mixture was poured into a plastic container that was $8 \mathrm{~cm}$ in diameter by $6 \mathrm{~cm}$ in height before gelling. In Fig. 2 we present the isotropic scattering spectra measured for the $\mathrm{TiO}_{2}$ suspensions with three different concentrations.

Using our reconstruction algorithm, we recovered the particle-size distributions for all three $\mathrm{TiO}_{2}$ suspensions, as shown in Fig. 3. The curves with the symbols denote the values reconstructed from the photon-migration measurements, and the shortdashed curve represents the size distribution obtained from the sedimentation measurement for comparison. As can be seen, excellent agreement between our results and those for the sedimentation was achieved. Since we used the concentrated suspensions (whereas a dilute solution was used in the sedimentation measurement) and a log-normal distribution in our reconstruction, errors in agreement between the results from photon migration and sedimentation can be expected. To reconstruct a typical particle-size distribution, as shown in Fig. 3, we needed only a few seconds on a SunSparc 5 workstation. We found that the inversion results were not very sensitive to the choice of the initial estimates of the reconstructed parameters $(a, b$, and $c)$, which were as much as $100 \%$ greater than the true parameters, depending of course on the optical-property measurements. Since measured data at 800 wavelengths between 400 and $800 \mathrm{~nm}$ were input into our reconstruction algorithm, the inverse solution procedure was quite stable, whereas the solution procedure was unstable when we used frequency-domain photon-migration techniques (measurements were made at only 15 wavelengths). ${ }^{3}$

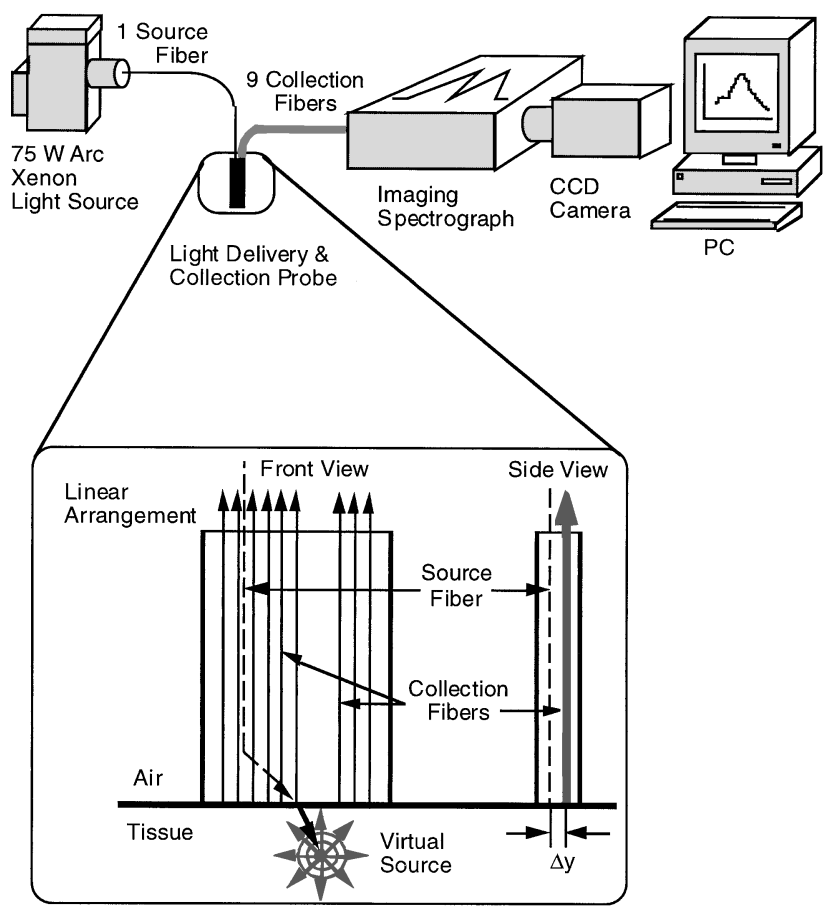

Fig. 1. Schematic of the experimental apparatus. White light was coupled to the oblique-incidence optical fiber probe. A source fiber delivered light to the gel phantoms at an angle of $45^{\circ}$, and the diffuse reflectance was collected by nine collection fibers. PC, personal computer. 


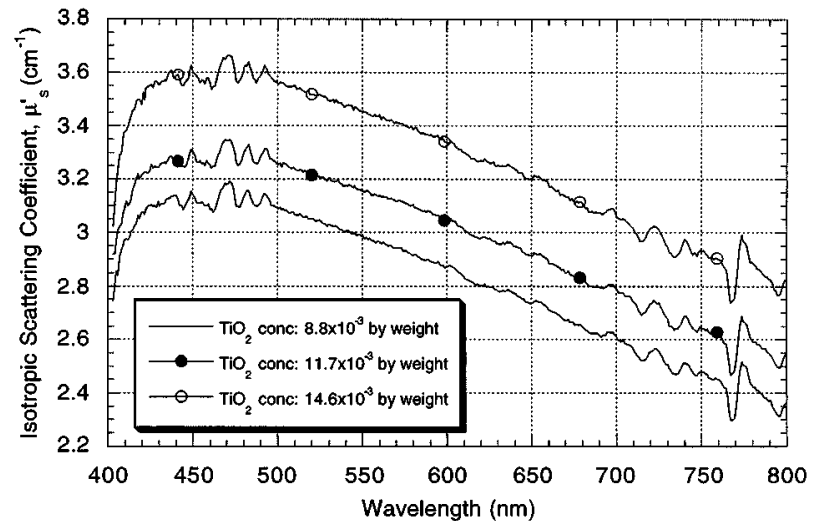

Fig. 2. Isotropic scattering spectra determined by the method of oblique-incidence reflectometry.

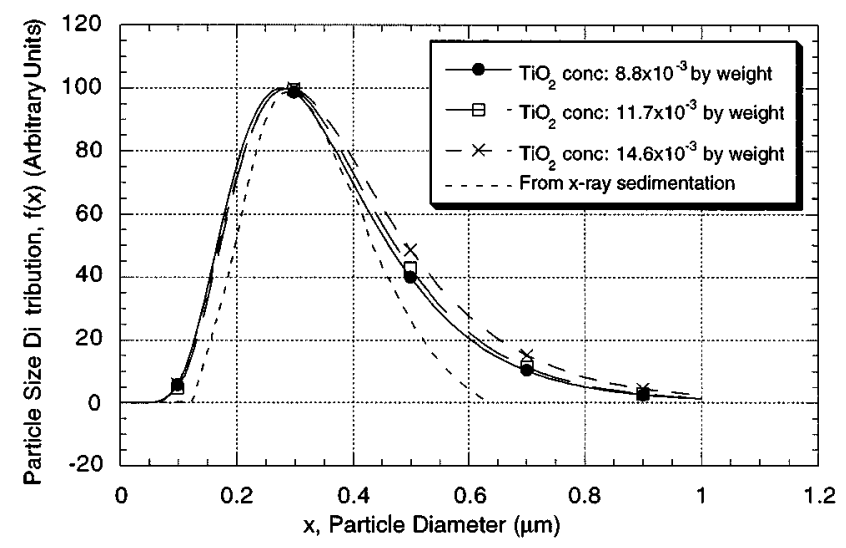

Fig. 3. Particle-size distribution as a function of diameter for the $\mathrm{TiO}_{2}$ samples as inverted from the diffuse reflectance measurements and as measured from the sedimentation.

It is also interesting to note that, although the shape of particles in $\mathrm{TiO}_{2}$ suspensions is not perfectly spherical, the spherical particulate-based Mie theory still provides accurate results from our inverse algorithm.
This accuracy could be due to the use of the hybrid regularization in our iterative optimization procedure, which provides a compensation mechanism for the geometric discrepancy that occurred in the theory.

In summary, we have used cw photon-migration measurements of isotropic scattering for particle sizing in concentrated suspensions. Our study shows that fast, simple $\mathrm{cw}$ photon-migration measurements in a wide wavelength range, coupled with an efficient inverse algorithm, can provide the particle-size distributions of concentrated suspensions in a matter of seconds. This result indicates the potential of the method described above for on-line process monitoring.

We acknowledge financial support from the Greenville Hospital System-Clemson University Biomedical Cooperative [for H. Jiang (HJ)], the Clemson University Research Grant Committee (HJ), the Whitaker Foundation [L. V. Wang $(\mathrm{LVW})]$, and the National Institutes of Health (grants R29CA68562 and R01CA71980 to LVW).

\section{References}

1. T. Allen, Particle Size Measurement (Chapman \& Hall, New York, 1990).

2. J. Wang and F. R. Hallet, Appl. Opt. 35, 193 (1996).

3. H. Jiang, J. Kao, and E. Sevick-Muraca, Appl. Opt. 36, 3310 (1997).

4. L.-H. Wang and S. L. Jacques, Appl. Opt. 34, 2362 (1995).

5. S.-P. Lin, L.-H. Wang, S. L. Jacques, and F. K. Tittel, Appl. Opt. 36, 136 (1997).

6. G. Marquez, L.-H. Wang, S.-P. Lin, J. A. Schwartz, and S. L. Thomsen, Appl. Opt. 37, 798 (1998).

7. H. Van de Hulst, Light Scattering by Small Particles (Dover, New York, 1983).

8. G. Bohren and D. Hoffman, Absorption and Scattering of Light by Small Particles (Wiley, New York, 1983).

9. E. L. Crow and K. Shimizu, Lognormal Distributions (Marcell Dekker, New York, 1988).

10. M. Lee, "New gel phantoms simulating optical properties of biological tissue," M. S. thesis (Texas A\&N University, College Station, Tex., 1997) . 